\section{Reassessment of patients with esophageal cancer after neoadjuvant therapy}
A. Das ${ }^{1}$, A. Chak $^{2}$
${ }^{1}$ Divisions of Gastroenterology, Mayo Clinic Scottsdale, AZ, USA
${ }^{2}$ Case Western Reserve University School of Medicine, Cleveland, $\mathrm{OH}$, USA

\section{Introduction}

Esophageal cancer is the eighth leading cause of cancer with worldwide estimates of more than 400,000 new cases and over 300,000 cancer related deaths annually [1]. Given the uniformly poor results achieved with surgical intervention alone, treatment at many centers has shifted to a multimodality approach that incorporates pre-operative neoadjuvant chemo-radiotherapy in patients with invasive disease who are potential candidates for subsequent surgical resection [2]. There are several rationales for this treatment strategy. First, down-staging may lead to improved surgical resectability. Second, the use of neoadjuvant therapy up front may prevent the systemic spread of cancer more effectively than post-operative chemo-radiotherapy, which is often deferred for several months to allow for surgical recovery. Third, chemo-radiotherapy (CRT) may be better tolerated preoperatively. Fourth, the tumor tissue may be better oxygenated prior to surgery leading to a higher kill of cancerous cells. Finally, a rather pessimistic argument in favor of neoadjuvant therapy is that sub-clinical metastatic disease may manifest itself in the preoperative period during which the neoadjuvant therapy is being administered and these patients may be spared extensive surgical resection [3]. Although neoadjuvant therapy is supported by sound clinical logic, results from available studies evaluating the role of neoadjuvant therapy in this setting are promising but still equivocal. While two large trials (the United Kingdom MAGIC trial [4] and the UK Medical research Council trial [5]) showed survival advantage with neoadjuvant chemotherapy, another large study from the US (the US Intergroup trial [6]) failed to show any major benefit. A meta-analysis from the Cochrane review group found improved 5-year survival in patients who underwent neoadjuvant chemotherapy. However, the improved survival could not be explained on the basis of proportion of patients undergoing resection or RO resection [7]. Similarly, large randomized trials that evaluated preoperative CRT with surgery against surgery alone and a subsequent metaanalysis failed to show impressive survival benefit with neoadjuvant CRT [8-11].

With such mixed results, it is clear that only a subgroup of patients with esophageal cancer potentially benefit from neoadjuvant therapy. Therefore, a focus of active research in this field is

Correspondence: Amitabh Chak, MD · Division of Gastroenterology · Wearn II Case Western Reserve University School of Medicine - 11100 Euclid Avenue Cleveland, OH, USA · Phone: 2168445386 - Fax: 2168448011 - E-mail: Amitabh.chak@case.edu

Bibliography: Endoscopy 2006; 38 (S1): S13-S17 @ Georg Thieme Verlag KG Stuttgart · New York · ISSN 0013-726X · DOI 10.1055/s-2006-946644 the identification of the subset of patients who respond to neoadjuvant therapy. From a clinical perspective, one would like to know before or early in the course of neoadjuvant therapy, which cancers are likely to respond. Ideally, if there were biomarkers, histologic characteristics, or morphologic criteria that directly allowed one to predict response to particular chemotherapeutic agents, then appropriate patients could be selected for neoadjuvant therapy. In lieu of such predictive markers, even if there was a method to detect response to neoadjuvant therapy early in the course of treatment, patients unlikely to respond could avoid further toxicity associated with the treatment. In the absence of a method for assessing response early during neoadjuvant treatment, even a method for restaging the tumor after completion of CRT could help guide decisions regarding subsequent curative surgery. Patients with a complete pathological response to induction therapy appear to have the best long term outcome [10-12]. Thus, a modality that accurately assessed response to treatment could allow stratification of patients according to the likelihood of further surgical benefit and could optimize the management of these patients.

\section{Assessment of response to neoadjuvant therapy}

It is known that simple clinical variables such as histological types and pre-treatment tumor stage do not reliably predict response to neoadjuvant therapy. While the role of biological markers such as level of expression of cycloxygenase 2 mRNA, survivin mRNA, tumor suppressor gene p53, proliferative marker Ki-67, epidermal growth factor and gene and protein expression profiling in predicting response to neoadjuvant therapy are being studied at an experimental level [13-15], in clinical practice the response to neoadjuvant therapy in these patients has either been assessed by morphological imaging such as computed tomography and endoscopic ultrasonography (EUS) or metabolic imaging such as fluorine 18 fluoro-deoxyglucose positron emission tomography, or a combination of these imaging tests.

\section{Computed tomography (CT) for restaging}

Several studies of computed tomography for assessing response after neoadjuvant therapy in patients with esophageal cancer have been published [16-18]. In a systematic review of published articles meeting strict inclusion criteria, Westerterp et al., calculated the sensitivity and specificity of CT in predicting pathological response to neoadjuvant therapy to range from 33 to $55 \%$ and $50 \%$ to $70 \%$, respectively [19]. This relatively poor discrimination between responders and non-responders is because CT is unable to distinguish between viable tumor from reactive changes such as scar tissue, necrosis, and edema. CT also cannot easily distinguish a hiatal hernia from a mass at the esophagogastric junction and likely has limited ability to distinguish tumor thickening from a collapsed or contracted esophagus. CT is particularly poor in differentiating the various $\mathrm{T}$ stages. It should however be noted that most studies have used single detector technology with $8-10 \mathrm{~mm}$ tomographic slices. In a recently published study using third generation or higher CT machines with $5 \mathrm{~mm}$ columnated cuts, the restaging accuracy of CT was reported to be $76 \%$ for T stage and $78 \%$ for nodal disease [20]. 
Endoscopic ulltrasonography (EUS) for restaging

EUS is currently the best modality for the initial regional, especially $\mathrm{T}$, staging of esophageal cancers. Of course, thoraco-abdominal CT is still a necessary part of pre-treatment staging because it detects distant metastatic disease, particularly in the liver and the lungs. It is not surprising that more than 10 published studies have also evaluated the role of EUS, if any, in the restaging of patients with esophageal cancer after neoadjuvant therapy [19]. The majority of these reports are single center experiences with limited, less than 100, patient numbers. Therefore, the results have been subject to the institutional expertise of the endosonographer. The more recent studies that attempted to correlate traditional EUS based $\mathrm{T}$ staging with pathologic surgical specimens have shown very poor accuracies, ranging from 29 to $59 \%[20-24]$.

EUS suffers from the same disadvantage common to all morphological imaging. It can not distinguish between viable tumor and post-necrosis residual inflammatory and scar tissue. This limitation is supported by the finding that reported accuracy of restaging with EUS after neoadjuvant therapy varies and is suboptimal. The wide variation in accuracy of restaging by EUS is partly explained by variations in the design and definitions and also, measured outcomes (pathological response vs. overall survival) used in some of these studies.

Given the number of reported studies consistently demonstrating poor accuracy of traditional EUS criteria for defining $\mathrm{T}$ stage following neoadjuvant therapy, some researchers have attempted to assess changes in tumor size by measuring tumor thickness or maximal cross sectional area before and after neoadjuvant therapy. These measurements are obviously indirect measures of tumor volume. As such, they are subject to some measurement error. Thickness is a single dimensional assessment and errors in measured thickness will be compounded to the third power when used to assess tumor volume; errors in measurements of area will be compounded by one order of magnitude when used as an indirect assessment of tumor volume.

A few studies have examined reduction in tumor thickness as a method for assessing neoadjuvant response. Bowrey et al. studied 17 esophageal cancer patients with EUS before and after CRT [24]. Although the T stage using traditional EUS criteria was unchanged in almost all patients, 10 of the 17 showed a reduction in the thickness of $2 \mathrm{~mm}$ or more. All four patients who had a complete pathologic response at resection were in this group. Swisher et al. obtained CT, EUS, as well as FDG-PET before and after CRT in 103 patients [25]. Their study found that standardized uptake value (SUV) of PET was the most accurate test for predicting survival but also noted that tumor thickness as measured by EUS was correlated with pathologic response. Both studies found that reduction in tumor thickness correlated with response but the reduction was only a few millimeters. This is because small changes in thickness can reflect large changes in volume.

Measurement of area is likely a more reliable method for assessing change in overall size. Willis et al in a study of 41 patients with esophageal cancer showed that positive response as assessed by measuring reduction in tumor area by EUS correlated well with pathological response [26]. A positive response on EUS was defined as a greater than $50 \%$ reduction in maximal tumor crosssectional area. The same group of investigators in a separate study showed that positive response on EUS, as defined by the maximal tumor cross sectional area also correlates with improved survival [27]. Other investigators have also shown that maximal cross sectional area correlated with survival and histological evidence of response [28]. It is, however, fair to note that correlation between tumor regression even when based on pathologic assessment has not always been found to correlate with survival.

Because patients with recalcitrant nodal disease after neoadjuvant therapy have markedly poor survival [29], it is important to assess for nodal response after neoadjuvant therapy and many surgeons will not consider curative resection in patients with persistent nodal disease. Reported accuracy of $\mathrm{N}$ staging by EUS also varies widely. Studies that did not incorporate EUS guided FNA for $\mathrm{N}$ staging reported suboptimal accuracy of EUS in $\mathrm{N}$ staging [21,22]. However, EUS guided FNA for confirmation of suspicious lymph nodes improves the accuracy of EUS N staging [20]. In general, with restaging by EUS, accuracy of N staging is thought to be higher than $\mathrm{T}$ staging. The overall sensitivity and specificity of EUS to predict response to neoadjuvant therapy from selected studies of poor to moderate methodological quality have been reported to range from 50 to $100 \%$ and $36 \%$ to $100 \%$, respectively [19].

Fluorine 18 fluoro-deoxyglucose (FDG) positron emission tomography (PET) for restaging

PET has rapidly become a non-invasive, whole body imaging method for the pre-operative staging of a variety of cancers. The increased glucose metabolism of malignant cells is imaged by the uptake of FDG a "radiotracer" in oncological PET studies. Unlike EUS, one of the basic advantages of FDG PET is its ability to image the whole body and quantify uptake in the entire volume of the primary tumor, thus measuring response of the primary tumor as well as metastatic disease in a single examination. Several studies have assessed the role of FDG-PET in the restaging of patients with esophageal cancer after neoadjuvant therapy.

Brucher et al reported the role of FDG-PET in 27 patients with squamous cell type of esophageal cancer who underwent neoadjuvant CRT [30]. Quantitative measurement (standardized uptake value or SUV) of tumor FDG uptake was correlated with histo-pathological response in the subsequently resected surgical specimen as well as survival. An ROC analysis showed that a threshold of $52 \%$ decrease of FDG uptake compared with baseline had a sensitivity and specificity of $100 \%$ and $55 \%$, respectively in predicting response to CRT. Also, patients with decrease in the FGD uptake of less than $52 \%$ had a significantly shorter median survival time compared with patients with an SUV decrease of more than $52 \%$ ( 8.8 vs. 22.5 months, $\mathrm{p}<0.0001$ ). The findings of the study were subsequently confirmed by other investigators $[31,32]$. While the study by Brucher et al assessed the PET response for the primary tumor only, subsequent investigators considered both primary tumor sites and nodal disease to define complete response to CRT. In a systematic review of published information, the overall joint sensitivity-specificity ( $Q$ point) values for FDG-PET was reported to be $85 \%$ ( $95 \% \mathrm{CI}, 77$ - $93 \%$ ) which was no different than that of EUS ( $86 \%, 95 \% \mathrm{CI}, 80-93 \%)$ and significantly better than that of CT $(54 \%, 95 \% \mathrm{CI}, 31-77 \%)$ [19]. 


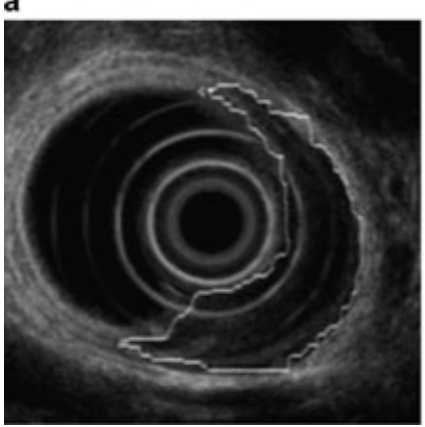

b

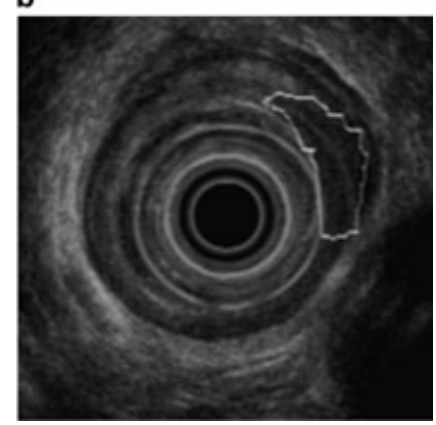

Fig. 1 EUS of patient with esophageal cancer (a responder) before (a) and after (b) chemoradiation with tracing of maximal cross-sectional tumor area shown. a

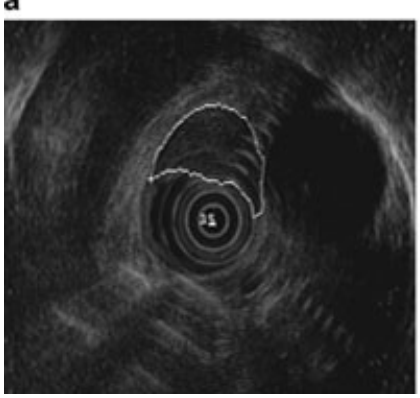

b

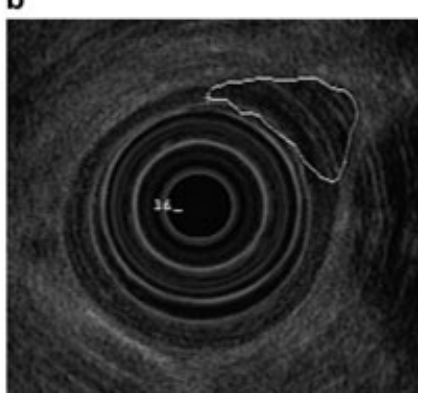

Fig. 2 EUS of patient with esophageal cancer (a nonresponder) before (a) and after (b) chemoradiation with tracing of maximal cross-sectional tumor area shown.

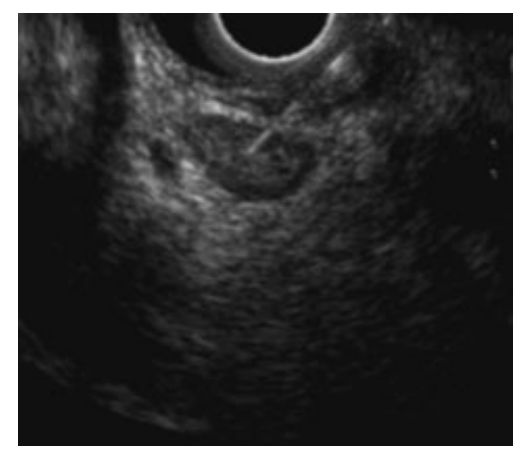

Fig. 3 EUS guided fine needle aspiration (EUS FNA) of a suspicious periesophageal lymph node performed using a linear echoendoscope.

\section{FGD PET vs. EUS in restaging}

It is increasingly clear that for restaging of patients with esophageal cancer who have undergone neoadjuvant treatment, EUS and FDG PET provide complementary information. The relative advantages with FDG PET scanning is that it is noninvasive, is feasible in most patients (unlike EUS where it is either not feasible or suboptimal in a significant proportion of patients [28]), provides opportunity for whole body imaging with a single test and, when integrated with CT as PET CT, provides both structural and metabolic information. FDG PET has high reproducibility and internal validity [33], can be used to assess early response and almost all published studies of FDG PET shows its ability to clearly differentiate responders and non-responders in terms of long term survival [34]. Also, unlike EUS, pre-CRT FDG-PET has been shown to correlate with response to CRT and is the only imaging modality that may identify a priori those patients who are likely to attain a major or even complete pathologic response to neoadjuvant treatment [32,34]. The disadvantages with FDG-PET are that in absence of tissue diagnosis, both false positive and false negative results are often misleading. The false positive results which lead to underestimation of effect of CRT are related to post CRT reactive inflammation with influx of leucocytes and scavenging macrophages in the tumor site. With application of sophisticated analytic models to interpret tumor uptake of FDG in terms of SUV measurements, the false positive rates may be lowered [34]. The false negativity of PET scans is due to the foci of residual cancer falling below the detection of threshold of the PET device and is a major concern particularly for assessment of nodal disease. Up to $20 \%$ of the adenocarcinomas of the gastroesophageal junction, particularly those with poor differentiation, have low FDG-avidity. In these patients FDG PET is not accurate for restaging after neoadjuvant therapy. FDG PET has higher false negative rates with cervical and abdominal lymph nodes compared to thoracic group of lymph nodes. Also, sensitivity for detecting small peritumoral lymph node is often low due to limited resolution of the PET device to differentiate it from the scatter effects seen in the area of primary tumor after CRT $[34,35]$. The unique advantage of EUS relative to other imaging modalities is its ability to obtain tissue aspirate for pathological assessment. Also, EUS is very accurate in identifying small peritumoral lymph nodes which are often below the threshold of detection of FDGPET. Only two published studies have directly compared the performance characteristics of different modalities for restaging of esophageal cancer after neoadjuvant therapy in the same patient population. In a prospective study reported by Cerfolio et al., 41 consecutive patients of esophageal cancer from a single center underwent restaging by CT, EUS with FNA and FDG-PET/CT after preoperative neoadjuvant CRT [19]. The accuracy of CT, EUS-FNA and FDG-PET/CT in distinguishing pathologic T4 disease from T1-T 3 stage was $76 \%, 80 \%$ and $80 \%$ respectively. The accuracy for nodal disease was $78 \%, 78 \%$ and $93 \%$. The authors concluded that FDG-PET/CT was more accurate than EUS-FNA and CT for predicting nodal status. PET was also better at identifying complete responders after neoadjuvant therapy. It is to be noted that in this study an integrated FDG-PET/CT scanner was used which may explain the relatively high negative predictive value of FDG-PET reported in this study. Also, for restaging, the authors used only traditional $\mathrm{T}$ staging by EUS and combined $\mathrm{T} 1$ to $\mathrm{T} 3$ disease in to one category on the basis of their argument that in their practice all T1-T3 disease persistent after CRT was treated by surgical resection if there was no nodal disease. They did not assess tumor size by EUS, which is the only EUS measure that has been found to be accurate for assessing neoadjuvant response. Swisher et al. retrospectively reviewed 103 patients who had PET, EUS, and CT performed before and after CRT [25]. Decreased wall thickness measured by EUS or CT and lower standardized uptake values (SUV) on PET correlated with pathologic response but post-CRT PET SUV $\geq 4$ was the best predictor of response. The PET SUV was also an independent predictor of survival.

No study to date has examined whether the combination of EUS measurement of cross sectional area, EUS-FNA, and follow-up PET measurements can improve the accuracy for identifying response to CRT. 
Approximately half of the patients with esophageal cancer will have no or minimal response to neoadjuvant CRT. Futile neoadjuvant treatment with its toxicity, morbidity and costs, both direct and indirect, could be avoided if these patients could be identified early after initiating CRT. Both CT and EUS are insensitive in this respect since structurally they can not distinguish between viable and non-viable tumor tissue. In our experience, the EUS accuracy for restaging using maximal cross sectional area is worse when the time interval between completion of CRT and restaging EUS is less than two weeks. On the other hand, FDG-PET has a unique advantage in this situation because of its ability to selectively identify only viable tumor tissue, which is metabolically intact. In an interesting study of 40 patients with esophageal cancer, Weber et al showed that performing FDG-PET scan at 2 weeks after initiation of cisplatin based poly-chemotherapy, a metabolic response (defined as $35 \%$ or more reduction in initial SUV) could be identified, which predicted eventual clinical response after completion of CRT with more than $90 \%$ sensitivity and specificity [36]. Moreover, patients without a metabolic response also had significantly shorter time to progression/recurrence and shorter overall survival. Similar data on the ability of FDG-PET to predict early response was also reported by Kroep at al in a smaller number of patients [37].

\section{Summary}

Currently there is no single optimal imaging modality for assessing response in patients with loco-regional and potentially resectable esophageal cancer who undergo neoadjuvant CRT. There are also no clear guidelines for making clinical decisions based on the results of re-staging imaging tests. If post-neoadjuvant assessment of response is to be used to guide subsequent therapeutic decisions, a combination of different imaging modalities may be needed. The choice will obviously vary with the local availability of resources and expertise. It is fairly clear that a conventional CT, particularly the single detector variety, has no role in restaging and should not be obtained. Ideally, if a FDG-PET integrated with CT is available, then a FDG-PET/ CT may provide useful information. If PET/CT shows suspected M1a or M1b disease, which is accessible to EUS FNA, then a EUS FNA could help establish the diagnosis of metastatic disease. If the PET/CT shows no evidence of metastatic disease but there is suggestion of residual tumor, particularly nodal disease, then EUS with FNA of suspicious lymph nodes could also be helpful. It is not known if a repeat EUS examination should be performed in patients with a completely negative FDG-PET. Prospective studies are still needed to determine the optimal time interval for performing FDG-PET after initiating CRT.

For assessment of response of primary tumor to treatment by EUS, traditional T staging is unacceptably inaccurate. A surrogate measure of tumor volume, maximal cross sectional area, is a better parameter for assessing response. For assessment of nodal response, use of more liberal criteria for performing EUS guided FNA could improve the nodal staging accuracy of EUS compared to that of FDG-PET. Finally, promising development in the field of multi-detector CT scanning and also, innovative use of $3 \mathrm{D}$ tech- nologies in the area of both CT and EUS imaging may revitalize the role of these modalities in assessment of response after CRT in patients with esophageal cancer but are still likely to be subject to difficulties in differentiating inflammation and scar from viable cancer. Use of biomarkers and alternative forms of imaging, such as endoscopic magnetic resonance imaging are at a rudimentary stage of development and are not ready for clinical applications in the near future.

\section{References}

${ }^{1}$ Parkin DM. International variation. Oncogene 2004 Aug 23; 23 (38): 6329-6340

${ }^{2}$ Kelsen DP. Multimodality therapy of local regional esophageal cancer. Semin Oncol. 2005 Dec; 32 (Suppl 9): S6-10

${ }^{3}$ Mooney MM. Neoadjuvant and adjuvant chemotherapy for esophageal adenocarcinoma. J Surg Oncol. 2005 Dec 1; 92 (3): 230-238

${ }^{4}$ Cunningham D, Allum WH, Stenning SP et al. Perioperative chemotherapy in operable gastric and lower esophageal cancer: Final results of a randomized, controlled trial (the MAGIC trial, ISRCTN 93793971) (abstract). J Clin Oncol 2005; 23: 308s

${ }^{5}$ Medical Research Council Esophageal Cancer Working Group. Surgical resection with or without preoperative chemotherapy in esophageal cancer: a randomized controlled trial. Lancet 2002; 359: 1727

${ }^{6}$ Kelsen DP, Ginsberg R, Pajak TF et al. Chemotherapy followed by surgery compared with surgery alone for localized esophageal cancer. $\mathrm{N}$ Engl J Med 1998; 339: 1979

${ }^{7}$ Malthaner R, Fenlon D. Preoperative chemotherapy for resectable thoracic esophageal cancer (Cochrane Review). Cochrane Database Syst Rev 2001; 1: CD001556

${ }^{8}$ Nygaard K, Hagen S, Hansen HS et al. Preoperative radiotherapy prolongs survival in operable esophageal carcinoma: a randomized, multi-center study of preoperative radiotherapy and chemotherapy - the second Scandinavian trial in esophagus cancer. World J Surg 1992; 16: 1104

${ }^{9}$ Le Prise E, Etienne PL, Meunier B et al. A randomized study of chemotherapy, radiation therapy, and surgery versus surgery for localized squamous cell carcinoma of the esophagus. Cancer 1994; 73: 1779

10 Bosset JF, Gignoux M, Triboulet JP et al. Chemoradiotherapy followed by surgery compared with surgery alone in squamous-cell cancer of the esophagus. N Engl J Med 1997; 337: 161

${ }^{11}$ Urschel JD, Vasan H. A meta-analysis of randomized controlled trials that compared neoadjuvant chemoradiation and surgery to surgery alone for resectable esophageal cancer. Am J Surg 2003; 185: 538

12 Walsh TN, Noonan N, Hollywood D et al. A comparison of multimodal therapy and surgery for esophageal adenocarcinoma. N Engl J Med 1996; 335: 462

${ }^{13}$ Xi H, Baldus SE, Warnecke-Eberz U, Brabender J, Neiss S, Metzger R, Ling FC, Dienes HP, Bollschweiler E, Moenig S, Mueller RP, Hoelscher AH, Schneider PM. High cyclooxygenase-2 expression following neoadjuvant radiochemotherapy is associated with minor histopathologic response and poor prognosis in esophageal cancer. Clin Cancer Res. 2005 Dec 1; 11 (23: $8341-8347$

${ }^{14}$ Warnecke-Eberz U, Hokita S, Xi H, Higashi H, Baldus SE, Metzger R, Brabender J, Bollschweiler E, Mueller RP, Dienes HP, Hoelscher AH, Schneider PM. Overexpression of survivin mRNA is associated with a favorable prognosis following neoadjuvant radiochemotherapy in esophageal cancer. Oncol Rep. 2005 Jun; 13 (6): 1241 - 1246

15 Miyazono F, Metzger R, Warnecke-Eberz U, Baldus SE, Brabender J, Bollschweiler E, Doerfler W, Mueller RP, Dienes HP, Aikou T, Hoelscher AH, Schneider PM. Quantitative c-erbB-2 but not c-erbB-1 mRNA expression is a promising marker to predict minor histopathologic response to neoadjuvant radiochemotherapy in esophageal cancer. $\mathrm{Br} \mathrm{J}$ Cancer. 2004 Aug 16; 91 (4): 666-672

${ }^{16}$ Walker SJ, Allen SM, Steel A, Cullen MH, Matthews HR. Assessment of response to chemotherapy in esophageal cancer. Eur J Cardiothorac Surg 1991; 5: 519-522

17 Griffith JF, Chan AC, Chow LT, Leung SF, Lam YH, Liang EY, Chung SC, Metreweli C. Assessing chemotherapy response of squamous cell esophageal carcinoma with spiral CT. Br J Radiol. 1999 Jul; 72 (859): 678 -684 
18 Jones DR, Parker LAJr, Detterbeck FC, Egan TM. Inadequacy of computed tomography in assessing patients with esophageal carcinoma after induction chemoradiotherapy. Cancer. 1999 Mar 1; 85 (5): 1026 - 1032

${ }^{19}$ Westerterp M, van Westreenen HL, Reitsma JB, Hoekstra OS, Stoker J, Fockens P, Jager PL, Van Eck-Smit BL, Plukker JT, van Lanschot JJ, Sloof GW. Esophageal cancer: CT, endoscopic US, and FDG PET for assessment of response to neoadjuvant therapy - systematic review. Radiology. 2005 Sep; 236 (3): $841-851$

${ }^{20}$ Cerfolio RJ, Bryant AS, Ohja B, Bartolucci AA, Eloubeidi MA. The accuracy of endoscopic ultrasonography with fine-needle aspiration, integrated positron emission tomography with computed tomography, and computed tomography in restaging patients with esophageal cancer after neoadjuvant chemoradiotherapy. J Thorac Cardiovasc Surg. 2005 Jun; 129 (6): 1232 - 1241

${ }^{21}$ Kalha I, Kaw M, Fukami N, Patel M, Singh S, Gagneja H, Cohen D, Morris J. The accuracy of endoscopic ultrasound for restaging esophageal carcinoma after chemoradiation therapy. Cancer. 2004 Sep 1; 101 (5): 940 - 947

22 Zuccaro GJr, Rice TW, Goldblum J, Medendorp SV, Becker M, Pimentel $\mathrm{R}$, Gitlin L, Adelstein DJ. Endoscopic ultrasound cannot determine suitability for esophagectomy after aggressive chemoradiotherapy for esophageal cancer. Am J Gastroenterol. 1999 Apr; 94 (4): 906 - 912

${ }^{23}$ Beseth BD, Bedford R, Isacoff WH, Holmes EC, Cameron RB. Endoscopic ultrasound does not accurately assess pathologic stage of esophageal cancer after neoadjuvant chemoradiotherapy. Am Surg. 2000 Sep; 66 (9): $827-831$

${ }^{24}$ Bowrey DJ, Clark GW, Roberts SA, Hawthorne AB, Maughan TS, Williams GT, Carey PD. Serial endoscopic ultrasound in the assessment of response to chemoradiotherapy for carcinoma of the esophagus. J Gastrointest Surg 1999 Sep-Oct; 3 (5): $462-467$

${ }^{25}$ Swisher SG, Maish M, Erasmus JJ, Correa AM, Ajani JA, Bresalier R et al. Utility of PET, CT, and EUS to identify pathologic responders in esophageal cancer. Ann Thorac Surg 2004 Oct; 78 (4): 1152 - 1160

${ }^{26}$ Willis J, Cooper GS, Isenberg G, Sivak MVJr, Levitan N, Clayman J, Chak A. Correlation of EUS measurement with pathologic assessment of neoadjuvant therapy response in esophageal carcinoma. Gastrointest Endosc. 2002 May; 55 (6): 655 -661

${ }^{27}$ Chak A, Canto MI, Cooper GS, Isenberg G, Willis J, Levitan N, Clayman J, Forastiere A, Heath E, Sivak MVJr. Endosonographic assessment of multimodality therapy predicts survival of esophageal carcinoma patients. Cancer. 2000 Apr 15; 88 (8): 1788-1795

${ }^{28}$ Hirata N, Kawamoto K, Ueyama T, Masuda K, Utsunomiya T, Kuwano $\mathrm{H}$. Using endosonography to assess the effects of neoadjuvant therapy in patients with advanced esophageal cancer. AJR Am J Roentgenol. 1997 Aug;169(2):485-491. Erratum in: AJR Am J Roentgenol 1998 Feb; 170 (2): 510

${ }^{29}$ Rice TW, Blackstone EH, Adelstein DJ, Zuccaro GJr, Vargo JJ, Goldblum JR, Rybicki LA, Murthy SC, Decamp MM. N1 esophageal carcinoma: the importance of staging and downstaging. J Thorac Cardiovasc Surg. 2001 Mar; 121 (3): $454-464$

${ }^{30}$ Brucher BL, Weber W, Bauer M, Fink U, Avril N, Stein HJ, Werner M, Zimmerman F, Siewert JR, Schwaiger M. Neoadjuvant therapy of esophageal squamous cell carcinoma: response evaluation by positron emission tomography. Ann Surg. 2001 Mar; 233 (3): 300-309

${ }^{31}$ Kato H, Kuwano H, Nakajima M, Miyazaki T, Yoshikawa M, Masuda N, Fukuchi M, Manda R, Tsukada K, Oriuchi N, Endo K. Usefulness of positron emission tomography for assessing the response of neoadjuvant chemoradiotherapy in patients with esophageal cancer. Am J Surg. 2002 Sep; 184 (3): 279-283

32 Flamen P, Van Cutsem E, Lerut A, Cambier JP, Haustermans K, Bormans G, De Leyn P, Van Raemdonck D, De Wever W, Ectors N, Maes A, Mortelmans L. Positron emission tomography for assessment of the response to induction radiochemotherapy in locally advanced esophageal cancer. Ann Oncol. 2002 Mar; 13 (3): 361 - 368

${ }^{33}$ Weber WA, Ziegler SI, Thodtmann R, Hanauske AR, Schwaiger M. Reproducibility of metabolic measurements in malignant tumors using FDG PET. J Nucl Med. 1999 Nov; 40 (11): 1771 - 1777

${ }^{34}$ Flamen P, Lerut T, Haustermans K, Van Cutsem E, Mortelmans L. Position of positron emission tomography and other imaging diagnostic modalities in esophageal cancer. Q J Nucl Med Mol Imaging. 2004 Jun; 48 (2): $96-108$

${ }^{35}$ van Westreenen HL, Westerterp M, Bossuyt PM, Pruim J, Sloof GW, van Lanschot JJ, Groen H, Plukker JT. Systematic review of the staging performance of $18 \mathrm{~F}-$ fluorodeoxyglucose positron emission tomography in esophageal cancer. J Clin Oncol. 2004 Sep 15; 22 (18): 3805-3812
${ }^{36}$ Weber WA, Ott K, Becker K, Dittler HJ, Helmberger H, Avril NE, Meisetschlager G, Busch R, Siewert JR, Schwaiger M, Fink U. Prediction of response to preoperative chemotherapy in adenocarcinomas of the esophagogastric junction by metabolic imaging. J Clin Oncol. 2001 Jun 15; 19 (12): $3058-3065$

${ }^{37}$ Kroep JR, Van Groeningen CJ, Cuesta MA, Craanen ME, Hoekstra OS, Comans EF, Bloemena E, Hoekstra CJ, Golding RP, Twisk JW, Peters $\mathrm{GH}$, Pinedo HM, Lammertsma AA. Positron emission tomography using 2-deoxy-2-[18F]-fluoro-D-glucose for response monitoring in locally advanced gastroesophageal cancer; a comparison of different analytical methods. Mol Imaging Biol. 2003 Sep-Oct; 5 (5): 337-346 